\title{
HUMANIZAÇÃO DO ATENDIMENTO NO SETOR DE RADIOLOGIA: DIFICULDADES E SUGESTÕES DOS PROFISSIONAIS DE ENFERMAGEM
}

Maria de Lourdes Custódio Duarte ${ }^{1}$, Adelita Noro ${ }^{2}$

RESUMO: Este estudo identificou as principais dificuldades e sugestões dos profissionais de enfermagem para uma prática humanizada. Trata-se de uma pesquisa qualitativa, realizada em 2011 no setor de Radiologia de um hospital privado no Rio Grande do Sul; foram participantes 10 profissionais de enfermagem. A análise dos dados deu-se através do referencial da análise temática, possibilitando a emergência das categorias dificuldades para uma prática humanizada e sugestões para um trabalho humanizado. Conclui-se que a assistência de enfermagem é pautada por cuidado complexo, individualizado, integral e humanizado, apesar das dificuldades. Sugere-se que as instituições de saúde estimulem e promovam reuniões e programas institucionais sobre o tema.

DESCRITORES: Humanização da assistência; Serviço hospitalar de radiologia; Equipe de enfermagem.

\section{HUMANIZATION OF THE ATTENDANCE IN THE RADIOLOGY SECTOR: NURSING PROFESSIONALS' DIFFICULTIES AND SUGGESTIONS}

\begin{abstract}
This study identified the nursing professionals' main difficulties and suggestions for humanized practice. It is qualitative research, undertaken in 2011 in the Radiology sector of a private hospital in Rio Grande do Sul; 10 nursing professionals participated. The analysis of the data occurred through the framework of thematic analysis, making possible the emergence of the categories difficulties for humanized practice and suggestions for humanized work. It is concluded that the nursing care is based on complex, individualized, integral and humanized care, in spite of the difficulties. It is suggested that the health institutions should encourage and promote institutional meetings and programs on the topic.

DESCRIPTORS: Humanization of care; Hospital radiology service; Nursing team.
\end{abstract}

\section{HUMANIZAÇÃO DO ATENDIMENTO NO SETOR DE RADIOLOGIA: DIFICULDADES E SUGESTÕES DOS PROFISSIONAIS DE ENFERMAGEM}

RESUMEN: Este estudio tuvo el objetivo de analizar las orientaciones de enfermería dadas a las gestantes acerca de las quejas clínicas más frecuentes durante la gravidez. Es un estudio descriptivo de abordaje cualitativo, realizado entre los meses de marzo y abril de 2011, con la participación de 14 gestantes registradas en una unidad básica de salud de Gurupi, Tocantins. Fue utilizado un guión de entrevista semiestructurado y los resultados fueron analizados por medio del análisis de contenido. Los principales síntomas mencionados fueron náuseas, vómitos, edema y lumbalgia; las orientaciones prestadas por el enfermero en la asistencia prenatal de poco riesgo fueron adecuadas y posibilitaron comprensión, por parte de las gestantes, de la sintomatología presentada. Fueron indicadas intervenciones pertinentes a las manifestaciones, a pesar de no se contemplar todas las intervenciones recomendadas.

DESCRIPTORES: Cuidado prenatal; Enfermería; Calidad da asistencia a la salud.

\footnotetext{
${ }^{1}$ Enfermeira. Mestre em Enfermagem. Doutoranda em Enfermagem pela Universidade Federal do Rio Grande do Sul. Professora do Curso de Graduação em Enfermagem da Universidade Federal do Pampa.

${ }^{2}$ Enfermeira do Serviço de Medicina Nuclear e Tomografia por emissão de pósitron do Hospital Mãe de Deus de Porto Alegre, Rio Grande do Sul.
} 


\section{INTRODUÇÃO}

Na busca de atendimento de qualidade aos usuários dos serviços de saúde, através de um trabalho pautado em uma equipe multidisciplinar com foco nas necessidades individuais e subjetivas dos sujeitos, foi intensificada, nos últimos anos, a discussão sobre humanização. Para que princípios como equidade, integralidade e participação social do usuário, entre outros, possam se efetivar, é necessário que as práticas de saúde sejam revistas, reconhecendo-se que o direito à saúde é uma questão de cidadania ${ }^{(1)}$. Nesse sentido, foi criada no Brasil a Política Nacional de Humanização $(\mathrm{PNH})^{(2)}$.

A humanização, para a $\mathrm{PNH}$, é um pacto, uma construção coletiva que só pode acontecer a partir da constituição de troca de saberes, através do trabalho em rede com equipes multiprofissionais, da identificação das necessidades, desejos e interesses dos envolvidos, do reconhecimento de gestores, trabalhadores e usuários como sujeitos ativos e protagonistas das ações de saúde e da criação de redes solidárias e interativas, participativas e protagonistas do Sistema Único de Saúde (SUS) ${ }^{(2)}$. Entende-se que a humanização deve perpassar todos os serviços de saúde, em seus mais variados setores, em todos os níveis de atenção. É na atenção terciária, que geralmente, se tem as maiores dificuldades para efetivar o cuidado humanizado, justamente por esses serviços serem de alta complexidade e trabalharem, principalmente, com tecnologias duras ${ }^{(3)}$.

Assim, destaca-se o setor de radiologia como um exemplo de serviço especializado que trabalha com tecnologia e maquinário de ponta. Esse serviço é extremamente complexo em virtude das atividades desenvolvidas e dos exames realizados, contribuindo para maior resolutividade diagnóstica de patologias clínicas e cirúrgicas, sendo um recurso cada vez mais utilizado $^{(4)}$.

Nesse sentido, a equipe de enfermagem surge como uma categoria imprescindível no setor de Radiologia, pois desenvolve atividades como administração da dose recomendada de radiofármaco; orientação quanto aos procedimentos a serem realizados; agendamento dos exames preliminares; controle e administração da medicação prescrita; orientação quanto à internação e alta; e atendimento de imediato às eventuais intercorrências clínicas ${ }^{(5)}$. Esses profissionais devem ser munidos de boa técnica e de habilidades para lidar com o ser humano em fase de vulnerabilidade frente à possibilidade ou certeza de uma doença, através do acolhimento, vínculo e escuta qualificada.
A motivação em realizar este estudo advém da observação do trabalho dos profissionais de enfermagem em um setor de radiologia, no qual atuam com equipamentos de alta tecnologia e onde muitas vezes exercem um trabalho mecanizado. A cobrança pela produtividade, entendida aqui como número de exames realizados ao dia, faz com que muitos profissionais esqueçam e de que todo exame envolve um ser humano, e que toda descoberta de uma doença envolve um sofrimento psíquico.

O relatado parece que é algo simples de ser feito, que deve ser natural, já que seres humanos cuidam de seres humanos. No entanto, observa-se a dificuldade de alguns profissionais chamarem o paciente pelo nome, percebê-lo como alguém que necessita de atenção e escuta, indo além da tecnologia proporcionada por uma máquina. O que se quer não é a negação da tecnologia, mas, sim, é a utilização de outras formas de perceber o paciente, indo ao encontro da PNH.

Somado a essas observações, salienta-se a escassez de estudos sobre a atuação da enfermagem na área de radiologia, e também de trabalhos sobre a humanização nessa área, o que demonstra a relevância do tema. Assim, constitui-se como problema de pesquisa: Qual a percepção dos profissionais de enfermagem sobre a humanização no setor de radiologia?

Os objetivos deste estudo foram identificar as principais dificuldades encontradas pelos profissionais da enfermagem para a realização da humanização no setor de radiologia e conhecer as sugestões dos profissionais para uma prática mais humanizada.

\section{MÉTODO}

Este estudo tem caráter exploratório-descritivo com abordagem qualitativa ${ }^{(6)}$ e foi realizado no setor de radiologia de um hospital privado do Rio Grande do Sul. Esse setor conta com as seguintes modalidades de exames por imagem: raios X; ecografia; mamografia; mamotomia; densitometria óssea; ressonância magnética; tomografia computadorizada; medicina nuclear, entre outros.

Os participantes do estudo compreenderam a totalidade de enfermeiros do setor (quatro) e de seis técnicos, sendo dois de cada turno de trabalho e que concordaram em participar do estudo, totalizando 10 entrevistados. O tipo da amostra foi intencional, por convite, tendo como critérios de inclusão: tempo mínimo de seis meses de vínculo com a instituição e desenvolver as atividades no setor de radiologia. A coleta dos 
dados ocorreu no período de agosto e setembro de 2011, por meio de entrevista individual semiestruturadas, consistindo de três questionamentos: Existe alguma dificuldade para efetivar a humanização do atendimento no setor de radiologia? Quais? Você teria alguma sugestão para a concretização da humanização nesse setor?

As entrevistas foram pré-agendadas e coletadas em uma sala reservada do setor de radiologia com tempo de duração em torno de 30 minutos. A pesquisa foi aprovada pelo Comitê de Ética do referido hospital, conforme Protocolo de número 456b/11, sendo considerados os aspectos éticos envolvendo seres humanos, em conformidade com o previsto na Resolução 196/96 do Conselho Nacional de Saúde ${ }^{(7)}$. Os participantes assinaram o Termo de Consentimento Livre e Esclarecido após terem sido informados sobre os objetivos da pesquisa e foram numerados de 1 a 10 , na ordem em que foram entrevistados, e denominados de $\mathrm{E}$ (enfermeiros) e $\mathrm{T}$ (técnicos de enfermagem).

Procedeu-se a leitura integral das entrevistas, adotando-se o referencial de análise temática para apreciação crítica do conteúdo, buscando-se encontrar os trechos significativos para a constituição dos temas abordados nas obras pesquisadas, em relação aos objetivos do estudo. Seguindo-se os passos de pré-análise e exploração do material foi possível a organização e leitura repetida do corpus de pesquisa. Posteriormente, procedeu-se ao tratamento e interpretação dos resultados obtidos, descritos em unidades de registro e de contexto, que permitiram o agrupamento de ideias relevantes em categorias ${ }^{(6)}$, representadas por dois eixos temáticos de discussão, a seguir apresentados.

\section{RESULTADOS}

\section{Dificuldades para uma prática humanizada}

Nessa categoria, são expostas as principais dificuldades enfrentadas pela equipe de enfermagem entrevistada para a efetivação da Humanização no setor de Radiologia. Foram elencados pelos entrevistados alguns itens que merecem destaque, tais como: 1) sobrecarga de trabalho; 2) atrasos na agenda versus tempo destinado para exames; 3) falta de comunicação entre os setores e profissionais; e 4) falta de profissionais qualificados para atuar na área de radiologia.

$\mathrm{O}$ grande número de exames realizados em um setor de Radiologia faz com que alguns profissionais sintam-se sobrecarregados, alegando que a demanda prejudica o atendimento humanizado, entendido pelos profissionais através da atenção e da conversa com o paciente, como recortes dos depoimentos:

A sobrecarga de trabalho, pela falta de profissionais, dificulta o atendimento humanizado. (E2)

Muito fluxo de pacientes, não conseguindo dar atenção que ele merece e que eu gostaria de dar porque a agenda está sempre lotada; e tem também falta de funcionários. Eu queria conversar com o paciente, mas eu sei que não posso, pois já tem outro paciente aguardando ali fora para entrar em sala. (T8)

$\mathrm{O}$ atraso no cumprimento da agenda de exames e o tempo destinado para a realização dos mesmos é percebido pela equipe de enfermagem como gerador de conflito e de estresse, tanto por parte dos profissionais quanto por parte dos pacientes, dificultando que o trabalho nesse setor seja entendido como humanizado:

O atraso acaba ficando estressante, gerando conflito, dificultando o atendimento humanizado. (T6)

Questões de tempo para cumprir a agenda e fazer todos os exames previstos para o turno gera estresse, mas depende de ti realizar um atendimento mais humanizado. (E4)

O hospital é uma instituição de saúde que deveria trabalhar de forma integrada para atender às necessidades dos pacientes que o procuram. No entanto o que muitas vezes percebe-se é um sistema desarticulado, no qual cada setor executa sua atividade sem comunicação com os demais. Essa desarticulação faz com que muitos procedimentos e exames sejam repetidos em decorrência da falta de comunicação entre os profissionais, como exemplificado:

O sistema do hospital não está organizado e integrado. Às vezes o paciente está na emergência e a punção que ele recebe não é adequadas para realizar um exame de tomografia. O que acontece é que ele é novamente puncionado no serviço. (T5)

A falta de comunicação entre os profissionais. O cuidado para não precisarmos repetir algum procedimento que poderiam ser resolvidos se tivesse comunicação entre as equipes. (T10)

A repetição de alguns procedimentos invasivos, 
como a punção venosa, é verbalizada pelos profissionais como uma ocorrência que interfere na efetivação da humanização no setor. Assim, a comunicação efetiva torna-se uma ferramenta extremamente importante para evitar que pacientes sejam expostos à repetição de procedimentos desnecessariamente.

Outro fator salientado pelos profissionais da equipe de enfermagem como um aspecto que dificulta a humanização no setor de Radiologia refere-se aos poucos profissionais capacitados para a realização de exames específicos, e que requerem qualificação diferenciada.

Poucos profissionais treinados para o atendimento de exames que exigem qualificação. (E4)

A partir do depoimento acima entende-se que, apesar da grande demanda de exames realizados no cotidiano do setor de Radiologia, há poucos profissionais com capacitação para a realização de alguns exames, o que pode contribuir para os atrasos, dificuldades ou grande demora para o agendamento.

$\mathrm{O}$ atendimento aos pacientes deve ser individualizado e personalizado, pois todos são diferentes e possuem suas particularidades no contexto da humanização. Dessa forma, os profissionais de enfermagem tentam adequar o cuidado prestado a cada paciente, apesar de sentirem estranhamento ao entrar em contato com pacientes que rejeitam alguma aproximação e carinho do profissional, como relatado:

O contato com o paciente é importante. Tem alguns pacientes fechados que não gostam que a gente se aproxime e faça carinho. Isso impede de tratar tão bem como gostaria para um atendimento humanizado. (T7)

Salienta-se que o paciente que procura o setor de Radiologia pode estar passando por algum processo de descobrimento de patologia e, nessa etapa, o sofrimento físico e mental pode levar a comportamento de rechaço de aproximação, tanto com os profissionais quanto com sua própria família. Cabe ao profissional ter discernimento e preparo para entender a etapa de vida pela qual essa pessoa está passando, e continuar a oferecer um atendimento humanizado, respeitando as necessidades de cada paciente.

\section{Sugestões para um trabalho humanizado}

Nesta categoria serão expostas as sugestões trazidas nas falas dos entrevistados para tornar o trabalho mais humanizado no serviço de radiologia. Dentre elas, destacam-se o número de profissionais, o cuidado integral e a consulta de enfermagem.

O aumento do número de profissionais foi percebido pelos entrevistados como uma sugestão para diminuir a sobrecarga dos que já atuam no setor, propiciando um atendimento de qualidade e humanizado aos pacientes:

Aumentar o número de profissionais no setor para que haja atendimento de qualidade e humanizado para os pacientes e profissionais. Nesse sentido, até já colocamos uma secretaria de posto para que pudéssemos estar mais próximos dos pacientes. Deixo sempre um técnico de enfermagem na sala de observação para que os pacientes sejam atendidos de forma integral e até mesmo um relógio de parede para que tenham a ideia do tempo que está aguardando para realizar o exame. (E1)

O profissional já descreve mudanças ocorridas no setor, como a criação de uma vaga de trabalho para uma secretária objetivando maior aproximação com pacientes. Descreve também, na qualidade de enfermeira e coordenadora da equipe de enfermagem, uma mudança na organização de processo de trabalho, ao deslocar um técnico de enfermagem para a sala de observação e a colocação de um relógio para que os pacientes tenham noção de tempo. Essas atitudes denotam uma discreta mudança na lógica do trabalho no setor de radiologia, possibilitando aos pacientes melhorias no atendimento e condições para que a equipe possa efetivar um cuidado mais humanizado no setor.

As ações da enfermagem devem constituir-se em um cuidado que vise a totalidade do indivíduo, ou seja, uma pessoa com sentimentos e com família, inserida em um contexto social que não pode ser descartado e, sim, utilizado em prol do seu cuidado, como exemplificado:

Deve-se olhar para o paciente e não apenas para a doença e exames. (E3)

A sugestão do entrevistado vai ao encontro da definição do cuidado integral na tentativa de superar a fragmentação do cuidado e de efetivar a humanização no setor, indo além da patologia e do exame que deve ser realizado.

A consulta de enfermagem é percebida como uma ferramenta que propicia melhor planejamento do atendimento e dos procedimentos que serão realizados durante a permanência do paciente no setor. Dessa 
forma, o melhor planejamento das ações da equipe de enfermagem qualificariam o cuidado, tornando-o humanizado, como relatado:

Procedimentos invasivos e mais demorados devem ser planejados previamente com uma consulta de enfermagem para que o paciente possa sentir-se mais tranquilo e integrado ao serviço. Assim, uma preparação bem planejada daria um melhor resultado. (E4)

Nessa categoria, evidencia-se a preocupação da equipe de enfermagem entrevistada em tornar mais humano o setor de radiologia, sendo expressas através de sugestões de melhorias, a partir das dificuldades observadas no cotidiano. Observa-se que as sugestões dos entrevistados são simples e exequíveis.

\section{DISCUSSÃO}

\section{Dificuldades para uma prática humanizada}

Evidencia-se nas falas dos entrevistados a vontade de tornar o atendimento mais humanizado no setor ,através de conversas com o paciente. No entanto, esses demonstram preocupação com as pessoas que aguardam a realização do exame e com a falta de funcionários.

Nesse contexto, muitos hospitais na atualidade são vislumbrados como empresas possuindo metas financeiras, levando-os, muitas vezes, a reduzir o quadro de funcionários para aumentar seus lucros. Essa atitude pode prejudicar a prestação de um atendimento de qualidade, pautado na atenção, no vínculo e na escuta, itens indispensáveis para um atendimento humanizado.

No setor de radiologia são realizados inúmeros exames, sendo destinado um tempo específico e muitas vezes restrito para sua realização. No entanto, o entrevistado entende que depende de cada profissional realizar um atendimento humanizado, independente do tempo que ele possui com o paciente.

A humanização é um processo simples e ao mesmo tempo complexo. Por vezes, muitos profissionais oferecem resistências, pois envolve mudanças de comportamento e condutas ${ }^{(8)}$. No entanto, cada profissional, cada equipe, cada instituição, terá seu processo singular de humanização. Dessa maneira, muitas podem ser as práticas humanizadas empregadas pelos profissionais, pois cada um faz sua própria leitura sobre o cuidado humanizado ${ }^{(9-10)}$.
Salienta-se que o trabalho no setor necessita de uma equipe multiprofissional, pois envolve múltiplos saberes. Tal equipe, geralmente, é composta de médico especialista em medicina nuclear, radiofarmacêutico, físico, enfermeiro, técnico em medicina nuclear e técnicos em radiologia e em enfermagem ${ }^{(5)}$.

Assim, a comunicação entre os profissionais é de extrema importância para um cuidado qualificado e humanizado. A informação do quadro clínico do paciente é importante, não só para que se adotem medidas padrão de precaução e prevenção de infecção hospitalar, que devem ser seguidas em qualquer situação, independentemente de confirmação diagnóstica, mas também para a prestação de um cuidado ético, humanizado e individualizado ${ }^{(4)}$.

Salienta-se que experiência de outra instituição demonstra que a contratação de profissionais qualificados e experientes, assim como os investimentos em treinamento e cursos de atualização profissional são medidas eficientes e que contribuem, significativamente, para a melhoria da qualidade dos serviços de radiodiagnóstico ${ }^{(11)}$.

Dessa forma, realizar periodicamente treinamentos e cursos de atualização profissional e implementar políticas e programas de qualidade e segurança radiológica nas instituições, incluindo a rotina de manutenção preventiva dos equipamentos, são medidas que qualificam o profissional e o cuidado prestado ${ }^{(11)}$.

$\mathrm{O}$ avanço tecnológico ocasionou o surgimento de outros tipos de exames na área da saúde, como tomografia computadorizada e ressonância magnética, gerando maior demanda de profissionais qualificados para trabalhar nesses setores ${ }^{(12)}$. Assim, entende-se que a qualificação dos profissionais não acontece na mesma velocidade com que ocorre o desenvolvimento de novas tecnologias, urgindo a necessidade de formação de profissionais para atuar no setor de radiologia.

Observa-se que, no cotidiano desses serviços, os gestores de saúde defrontam-se com crescentes dificuldades no desenvolvimento da prática da equipe de saúde pela utilização de procedimentos e tratamentos complexos, que exigem estrutura física adequada, pessoal capacitado e materiais cada vez mais modernos. Pois, os níveis de complexidade tecnológica refletem a natureza das tarefas a serem executadas para um sujeito também complexo ${ }^{(11,13)}$. Dessa forma, entende-se que a subjetividade do paciente e do profissional estão constantemente em interação com a complexidade do avanço tecnológico, influenciando na humanização do cuidado no setor de Radiologia. 


\section{Sugestões para um trabalho humanizado}

$\mathrm{O}$ atendimento integral contempla um olhar para o sujeito-usuário dentro da lógica de atendimento que considere o cuidado nas mais diversas dimensões do ser humano ${ }^{(14)}$. Esse cuidado deve ser um processo dinâmico e intencional para atender às necessidades humanas, um valor que pressupõe sensibilidade, afetividade, zelo, atenção, solidariedade e compromisso entre o ser cuidador e o ser cuidado ${ }^{(15)}$.

A consulta de enfermagem no setor de Radiologia tem como objetivo prestar assistência de enfermagem personalizada, sistematizada, facilitando a identificação de problemas de saúde/doença, a execução e a avaliação dos cuidados que contribuam para a promoção, proteção, recuperação e reabilitação de sua saúde ${ }^{(16)}$. Adotar o processo de enfermagem favorece a autonomia profissional e a tomada de decisões, contribuem para a visibilidade profissional e para a ciência da Enfermagem $^{(17)}$. Sua realização envolve uma sequência de ações que qualificam o cuidado, possibilitando ao profissional um espaço para atuar de forma humanizada a partir do encontro do profissional e o paciente. Nesse cenário, tematizar a humanização da assistência possibilita levantar questões fundamentais que podem auxiliar na construção das políticas em saúde. Humanizar é, deste modo, ofertar atendimento de qualidade, articulando os avanços tecnológicos com o acolhimento, com melhorias dos ambientes de cuidado e das condições de trabalho dos profissionais ${ }^{(2)}$. Assim, entende-se a necessidade de treinar e capacitar a equipe, não somente em assuntos técnicos, mas humanos, principalmente em momentos de vulnerabilidade do paciente, individualizando e humanizando o cuidado.

\section{CONSIDERAÇÕES FINAIS}

Ao longo deste estudo percebemos que a compreensão de humanização permeia o cotidiano da equipe de enfermagem entrevistada, orientando suas práticas, sendo entendidas através de suas dificuldades e sugestões para a efetivação da humanização no setor de radiologia.

A equipe de enfermagem percebe que a tecnologia é importante ferramenta no processo saúde/doença. Dessa maneira, as instituições de saúde devem estimular o cuidado humanizado como fator indispensável no cotidiano de quem cuida, além de promover programas institucionais sobre o tema.

Portanto, espera-se, que os dados deste estudo contribuam para a construção de novos trabalhos abrangendo a temática, e que os profissionais da equipe de enfermagem que atuam em setores de radiologia possam beneficiar-se e utilizá-los em seu cotidiano. Enfatiza-se a importância de mais estudos sobre esses setores, tendo em vista a escassez de produções, o que promoverá maior conscientização das organizações e dos investigadores para a humanização nesses serviços.

\section{REFERÊNCIAS}

1. Ministério da Saúde (BR). Secretaria de Assistência à Saúde. Programa Nacional de Humanização da Assistência Hospitalar [Internet]. Brasília; 2001 [acesso em 02 fev 2011]. Disponível em: http://bvsms.saude.gov. br/bvs/publicacoes/pnhah01.pdf.

2. Ministério da Saúde (BR). Política Nacional de Humanização: Humaniza SUS [Internet]. Brasília; 2005 [acesso em 11 fev 2011]. Disponível: http://portal.saude. gov.br/saude/area.cfm?id_area $=389$.

3. Mehry EE, Magalhães Júnior HM, Rimoli J, Franco TB, Bueno WS, organizadores. O trabalho em saúde: olhando e experienciando o SUS no cotidiano. São Paulo: Hucitec; 2004.

4. Espíndola KKL, Ramos IC, Leitão IMTA. Medidas de prevenção e controle de infecção: percepção e conhecimento dos técnicos em radiologia. Cienc. cuid. saude. [Internet] 2008;7(3) [acesso em 10 mar 2012]. Disponível: http://periodicos.uem.br/ojs/index.php/ CiencCuidSaude/article/view/6488/3856.

5. Flôr RC, Gelbcke FL. Tecnologias emissoras de radiação ionizante e a necessidade de educação permanente para uma práxis segura da enfermagem radiológica. Rev. bras. enferm. [Internet] 2009;62(5) [acesso em 25 ago 2012]. Disponível: http://dx.doi.org/10.1590/S0034-71672009000500021.

6. Minayo MCS. O desafio do conhecimento: pesquisa qualitativa em saúde. $10^{\mathrm{a}}$ ed. São Paulo: Hucitec; 2007.

7. Ministério da Saúde (BR). Conselho Nacional de Saúde. Resolução 196, de 10 de outubro de 1996: diretrizes e normas regulamentadoras de pesquisas envolvendo seres humanos. Brasília; 1996.

8. Martins MCF. Humanização das relações assistenciais de saúde: a formação do profissional de saúde. São Paulo: Casa do Psicólogo; 2001.

9. Deslandes SF, organizadora. Humanização dos cuidados em saúde: conceitos, dilemas e práticas. Rio de Janeiro: Fiocruz; 2006. 
10. Silva CRRG, Rodrigues VMCP. O que dizem os pacientes dos serviços privados de Radiologia, Portugal. Saude soc. [Internet] 2011;20(2) [acesso em $18 \mathrm{abr}$ 2012]. Disponível: http://dx.doi.org/10.1590/S010412902011000200014.

11. Santos Junior BJ, Hinrichsen SL, Lira C, Vilella TA. Riscos ocupacionais em centros de radio diagnóstico. Rev. enferm. UERJ. [Internet] 2010;18(3) [acesso em 23 set 2012]. Disponível: http://www.facenf.uerj.br/v18n3/ v18n3a05.pdf.

12. Flôr RC, Kirchhof ANC. Uma prática educativa de sensibilização quanto à exposição a radiação ionizante com profissionais de saúde. Rev. bras. enferm. [Internet] 2006;59(3) [acesso em 5 mar 2012]. Disponível: http:// dx.doi.org/10.1590/S0034-71672006000300005.

13. Brand CI, Fontana RT, Santos AVS. A saúde do trabalhador em radiologia: algumas considerações. Texto Contexto Enferm. [Internet] 2011;20(1) [acesso em 12 ago 2012]. Disponível: http://dx.doi.org/10.1590/ S0104-07072011000100008.

14. Duarte MLC, Noro A. Humanização: uma leitura a partir da compreensão dos profissionais da enfermagem. Rev. Gaúcha Enferm. [Internet] 2010;31(4) [acesso em 30 ago 2012]. Disponível: http://dx.doi.org/10.1590/ S1983-14472010000400011.

15. Mostardeiro SCTS, Pedro ENR. O cuidado de enfermagem em situações de alteração da imagem facial. Rev. Gaucha Enferm. [Internet] 2011;32(2) [acesso em 12 ago 2012]. Disponível: http://dx.doi. org/10.1590/S1983-14472011000200012.

16. Campos RMC, Ribeiro CA, Silva CV, Saparolli ECL. Consulta de enfermagem em puericultura: a vivência do enfermeiro na Estratégia de Saúde da Família. Rev Esc Enferm USP. [Internet] 2011;45(3) [acesso em 16 ago 2012]. Disponível: http://dx.doi.org/10.1590/S008062342011000300003 .

17. Santana LL, Mazza VA, Taube SAM, Sarquis LMM. Diagnóstico e intervenções de enfermagem em unidade de pronto atendimento à luz das necessidades humanas básicas. Cogitare enferm. [Internet] 2011;16(4) [acesso em 12 ago 2012]. Disponível: http://ojs.c3sl.ufpr.br/ojs2/ index.php/cogitare/article/view/25436/17057. 